ISSN 0258-7122 (Print), 2408-8293 (Online)

Bangladesh J. Agril. Res. 42(4): 715-724, December 2017

\title{
INVESTIGATION ON GENETIC CONTROL FOR YIELD AND YIELD CONTRIBUTING TRAITS IN ADVANCED GENERATION OF MAIZE (Zea mays L.)
}

\author{
S. BEGUM ${ }^{1}$, M. AmIRUZZAMAN ${ }^{2}$, A. AHMED ${ }^{3}$, S. H. OMY ${ }^{4}$ \\ AND M. M. ROHMAN ${ }^{5}$
}

\begin{abstract}
Twenty five cross progeny of maize developed by Plant Breeding Division of BARI were evaluated by determining general combining ability (GCA) and specific combining ability (SCA) effects following line $\times$ tester design involving five female lines and five testers for grain yield and other yield contributing traits. Highly significant genotypic variances specified inclusive inconsistency existed among the genotypes. None of the lines showed significant GCA effects for all the characters, but the line Pac-60/S $\mathrm{S}_{4}-3$ and Pac-60/S $\mathrm{S}_{4}-9$ showed negative GCA effect for days to $50 \%$ tasseling and silking which is desirable to develop early variety. Additionally, the line Pac-60/ $/ \mathrm{S}_{4}-9$, Pac-60/ $\mathrm{S}_{4}-18$ and Pac-60/S $4-21$ showed negative GCA effect for plant and ear height which is also desirable to develop dwarf variety. Nevertheless, none of the cross showed significant SCA effect for any character studied, but crosses Pac-60/S 4 - $21 \times$ BIL-113, Pac-60/S 4 $21 \times U t n / \mathrm{S}_{4}-15, \quad \mathrm{Pac}-60 / \mathrm{S}_{4}-18 \times \mathrm{Utn} / \mathrm{S}_{4}-8, \quad \mathrm{Pac}-60 / \mathrm{S}_{4}-3 \times \mathrm{BIL}-113, \quad \mathrm{Pac}-60 / \mathrm{S}_{4}-$

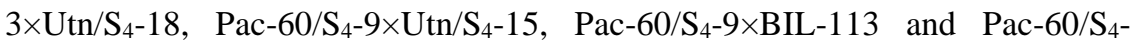
$21 \times \mathrm{Utn} / \mathrm{S}_{4}-10$ had higher yield with positive SCA effects. However, considering yield data along with lodging percentage, five crosses namely $\mathrm{Pac}-60 / \mathrm{S}_{4}$ $21 \times$ BIL-113, Pac-60/S $4_{4}-21 \times U$ tn $/ \mathrm{S}_{4}-15, \quad \mathrm{Pac}-60 / \mathrm{S}_{4}-21 \times \mathrm{Utn} / \mathrm{S}_{4}-10 \quad \mathrm{Pac}-60 / \mathrm{S}_{4}-$ $9 \times \mathrm{Utn} / \mathrm{S}_{4}-15$ and Pac-60/S 4 -3 $\times$ BIL-113 showed better performance.
\end{abstract}

Keywords: Line $\times$ tester, General combining ability, Specific combining ability.

\section{Introduction}

Maize is an important cereal crop in the world. Worldwide maize production was 960.73 million metric tons in 2015-16 (www.worldcornproduction.com). It grows over a wide range of geographical and environmental conditions extending from $58^{\circ} \mathrm{N}$ to $40^{\circ} \mathrm{S}$. Over 200 million people in developing countries like Asia, Latin America and Africa consume maize as a staple food (Chandel and Mankotia, 2014). Nowadays maize is globally well-known as a strategic food and feed crop that offers an enormous amount of protein and energy for humans and livestock as well. In unindustrialized countries maize is usually cast-off as food, while in the advanced world, it is used extensively as a major source of carbohydrate in animal feed. Industrially, maize is used to produce alcohol, starch, pulp abrasive, and oil in the pharmaceutics and recently for fuel production (Acharya and Young, 2008). In Bangladesh maize is extensively used

${ }^{1-5}$ Plant Breeding Division, Bangladesh Agricultural Research Institute, Gazipur-1701, Bangladesh. 
as poultry feed. The total maize cultivated area in Bangladesh was 3.96 million hectare, production was 27.59 million metric tons and national average yield was 6.97 ton per hectare in 2015-16 (DAE, 2017).

Maize retains enormous natural and inherited diversity which implicit the curiosity of geneticists and plant breeders for the improvement of this crop. In fact, maize has been subjected to broad genetic studies rather than any other crop (Hallauer and Miranda, 1988). One of the most important criteria for identifying high yielding hybrid is the information about parents' genetic structure and their combining ability (Ceyhan, 2003). Combing ability is the relative ability of a genotype to pass on its desirable performance to its offspring. Combining ability doesn't only offer the understanding of hereditary of quantitatively inherited behaviors but also provides crucial information about the selection of parents which creates better segregants. Obviously, line $\times$ tester analysis technique which suggested by Kempthorne (1957) is one of the prevailing tools offered to assess general and specific combining ability effects and assistances in picking desirable parents and crosses. The success of this technique depends mainly upon the nature of tester used in the evaluation. The suitable tester should include easiness in habit, deliver information that properly classifies the merit of lines and maximizes the genomic gain (Hallauer, 1975; Menz et al., 1999).

Line $\times$ tester analysis method developed by Kempthorne (1957) has been widely used by plant breeders throughout the world to generate reliable information on the general and specific combining ability effects of large number of parents and their hybrid combinations. Afterwards, this method has been widely used in maize by several workers and continues to be applied in quantitative genetic studies in maize (Rawlings and Thompson, 1962; Joshi et al., 2002; Sharma et al., 2004; Rahman et al., 2013; Amin et al., 2014; EL-Hosary, 2014; Chandel and Mankotia 2014; Kamara et al., 2015 and Liaqat et al., 2015). Thus, this method certainly can help us by providing appropriate information on grouping of materials to different heterotic patterns and also by estimating the type of gene action involved in the expression of yield and yield contributing traits. In this study, five advanced lines were used as testers which never been reported as testers before. Therefore, the genetic makeup of these testers was unknown. However, the objectives of the current study were to assess the female lines and identify superior one or more lines, compare different testers to find out most suitable one(s) and estimate the combining ability of the lines and testers.

\section{Materials and Methods}

A total of 25 crosses were made through line $\times$ tester design during rabi, 201314. The $25 F_{1}$ s, five $S_{4}$ parental lines (Pac-60/S $S_{4}-3$, Pac-60/S $S_{4}-4$, Pac- $60 / S_{4}-9$, Pac-60/S $\left.\mathrm{S}_{4}-18, \mathrm{Pac}-60 / \mathrm{S}_{4}-21\right)$ and five testers $\left(\mathrm{Utn} / \mathrm{S}_{4}-8, \mathrm{Utn} / \mathrm{S}_{4}-10, \mathrm{Utn} / \mathrm{S}_{4}-15\right.$, $\mathrm{Utn} / \mathrm{S}_{4}-18$, BIL-113) were grown in an alpha lattice design with two replications with a spacing of $60 \mathrm{~cm} \times 20 \mathrm{~cm}$ between rows and hills, respectively at BARI, 
Gazipur during rabi, 2014-15. Seeds were sown on $1^{\text {st }}$ week of November, 2014. One border row was kept at each end of the replication to curtail the edge effect. Fertilizers like N, P, K, S, Zn and B were applied @ 250, 55, 110, 40, 5 and $1 \mathrm{~kg}$ $\mathrm{ha}^{-1}$ respectively. The observations were recorded on ten randomly selected competitive plants in each replication for quantitative characters viz. days to $50 \%$ tasselling, days to $50 \%$ silking, plant height $(\mathrm{cm})$, ear length $(\mathrm{cm}), 1000$ grain weight $(\mathrm{g})$, yield (ton/ha). Lodging data was recorded and calculated as per cent. General Combining ability (GCA) and Specific Combining ability (SCA) were analyzed as per the method given by Kempthrone (1957).

\section{Results and Discussion}

Results pertaining to yield and yield contributing traits can be depicted below. Analysis of variance for combining ability was carried out for yield and other characters and the mean sum of squares were presented in Table 1. The analysis of variance revealed highly significant differences among the genotypes for all the characters studied except 1000 grain weight indicating sufficient genetic variability existed among the genotypes for these traits. Highly significant variances were observed among parents for plant height and ear height. Highly significant differences also showed among interactions of parent $\times$ crosses for all the traits indicated wide range of variability existed among them. Variability between crosses were highly significant for days to tasseling, days to silking, plant height and ear height, thus considerable amount of average heterosis were reflected in cross combinations for those traits. Lines had significant differences for days to tasseling, plant height and ear height; tester had significant differences for days to tasseling and days to silking. However, an extensive variation was not found in line $\times$ tester for any character under studied (Table 1).

A comparison of the magnitude of variance components due to GCA and SCA authorizes the gene action in governing the appearance of traits. Higher estimation of dominance variance $\left(\sigma^{2} \mathrm{SCA}\right)$ was observed as compared to additive variance $\left(\sigma^{2} \mathrm{GCA}\right)$ for all the characters except days to $50 \%$ tasseling (Table 1 ) probably due to predominance of non-additive gene action, suggesting the scope of improvement of these characters through heterosis breeding. Similar nonadditive gene action was also reported by Suneetha et al., (2000) for days to 50 per cent tasseling and days to 50 per cent silking, Kanagarasu et al., (2010) and Kumar et al., (2014) for grain yield, cob length, plant height, ear height, 1000 grain weight, grain rows per cob, days to 50 percent tassel and days to 50 percent silk and Ali et al., (2012) for number of grain rows per cob and 100-grain weight, Kumar et al., (2012) and Ahmed et al., (2015) for plant height, days to $50 \%$ tasseling, days to $50 \%$ silking, cob length, cob girth, number of grain rows per cob, number of grains per row, 1000-grain weight and grain yield in maize in their study. Singh and Singh (1998) also reported non-additive gene action for plant height, ear length, kernel rows, 1000 grain weight and yield in maize. 


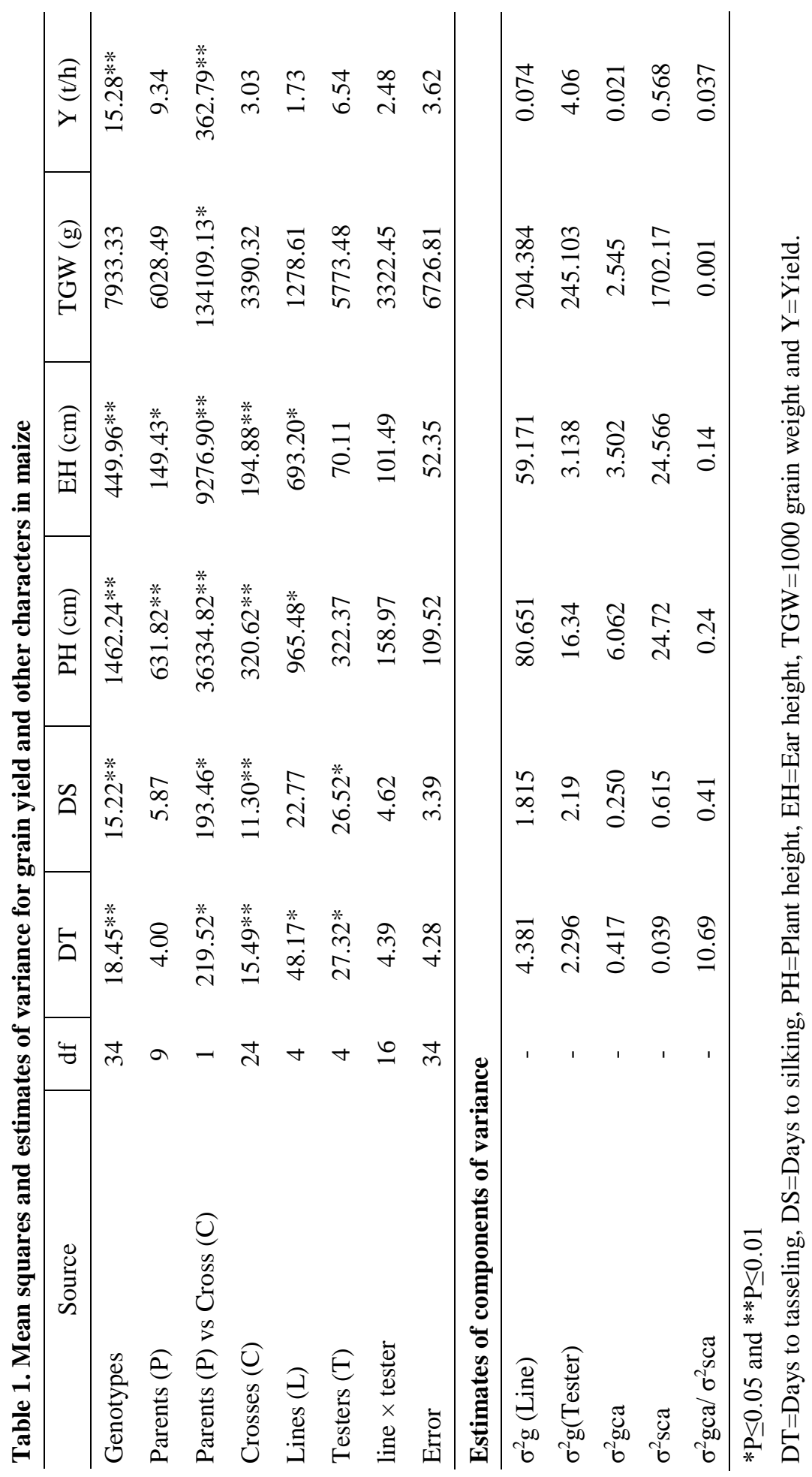


The contribution of lines, testers and their interactions to total variances were presented in Table 2. The proportional contribution of lines in days to tasseling, plant height and ear height was higher than tester and interaction suggested the maximum contribution of lines for these characters. However, the contributions of lines for 1000 grain weight and yield were lower than the interactions to total variances. This suggested female parents failed to contribute the maximum to total variance for these two characters in maize. Testers contributed higher than lines in days to $50 \%$ silking. Line $\times$ tester interaction donated the maximum for grain weight and yield to total variance.

Table 2. Proportional contribution of lines, testers and their interactions to total

\begin{tabular}{l|c|c|c|c|c|c}
\multicolumn{6}{c}{ variance in maize } \\
\hline Source & DT & DS & PH $(\mathbf{c m})$ & EH $(\mathbf{c m})$ & TGW $(\mathbf{g})$ & $\mathbf{Y ~ ( t / h )}$ \\
\hline Line & 51.840 & 33.599 & 50.188 & 59.286 & 6.286 & 9.534 \\
Tester & 29.402 & 39.132 & 16.758 & 5.996 & 28.382 & 35.957 \\
line $\times$ tester & 18.758 & 27.269 & 33.055 & 34.718 & 65.332 & 54.509 \\
\hline
\end{tabular}

$\mathrm{DT}=$ Days to tasseling, DS=Days to silking, $\mathrm{PH}=\mathrm{Plant}$ height, EH=Ear height, TGW=1000 grain weight and $\mathrm{Y}=$ Yield

\section{General combining ability}

Selection of parents with good general combining ability is a prime requisite for any successful breeding program especially for heterosis breeding. The general combining ability effects and per se performance of parents are presented in Table 3. The GCA effects of parents designated that most of the lines did not express significant desirable GCA effect for the traits. However, line Pac-60/S ${ }_{4}-3$ and Pac-60/S $4_{4}-9$ and tester $\mathrm{Utn} / \mathrm{S}_{4}-10$ and BIL-113 exhibited negative GCA effect for days to $50 \%$ tasseling and silking which suggested that they might contribute for earliness. For plant height, line Pac-60/S $4-9$, Pac-60/S ${ }_{4}-18$ and Pac-60/S $4-21$ and tester Utn $/ \mathrm{S}_{4-}$ 18 and BIL-113 had negative GCA with shorter plant height suggested these lines could be utilized for evolving dwarf variety. Pac-60/S4-21 showed significant negative GCA effect for ear height signifying it would be subsidized to develop short ear heighted plant. Line Pac $-60 / \mathrm{S}_{4}-3$ and tester $\mathrm{Utn} / \mathrm{S}_{4}-8, \mathrm{Utn} / \mathrm{S}_{4}-15$ and BIL-113 was found to have positive GCA effects for both 1000 grain weight and yield (Table 3) which suggested that it could be utilized for developing high yielding variety. The lines with high GCA effects demonstrating additive gene action in inheritance of characters may be employed in hybridization program to expand a specific trait through transgressive segregation. 
Table 3. General combining ability (GCA) effects and mean of parents for grain yield and other characters in maize

\begin{tabular}{|c|c|c|c|c|c|c|c|}
\hline \multirow{2}{*}{$\begin{array}{c}\text { Sl. } \\
\text { NO. }\end{array}$} & \multirow[t]{2}{*}{ Parents } & \multicolumn{2}{|c|}{ DT } & \multicolumn{2}{|c|}{ DS } & \multicolumn{2}{|c|}{ PH (cm) } \\
\hline & & GCA & mean & GCA & mean & GCA & mean \\
\hline \multicolumn{8}{|l|}{ Line } \\
\hline 1 & Pac-60/S $4-3$ & -2.68 & 92.5 & -2.42 & 97.5 & $10.84 * *$ & 115.63 \\
\hline 2 & $\mathrm{Pac}-60 / \mathrm{S}_{4}-4$ & 1.02 & 91.5 & 0.58 & 97 & 7.75 & 93.50 \\
\hline 3 & Pac-60/S $4-9$ & -1.68 & 91 & -0.12 & 98.5 & -1.10 & 103.8 \\
\hline 4 & Pac-60/S $4-18$ & 2.82 & 93.5 & 1.68 & 97.5 & -3.54 & 111.33 \\
\hline \multirow[t]{3}{*}{5} & $\mathrm{Pac}-60 / \mathrm{S}_{4}-21$ & 0.52 & 91.5 & 0.28 & 96.5 & $-13.96 * *$ & 101.00 \\
\hline & $\mathrm{SE}_{\left(g_{i}\right)}$ & 0.654 & - & 0.582 & - & 3.31 & \\
\hline & $\mathrm{SE}_{(} g_{i-} g_{j)}$ & 0.925 & - & 0.823 & - & 4.68 & \\
\hline \multicolumn{8}{|c|}{ Tester } \\
\hline 1 & $\mathrm{Utn} / \mathrm{S}_{4}-8$ & 0.62 & 95.0 & 0.28 & 100 & 1.69 & 105.8 \\
\hline 2 & $\mathrm{Utn} / \mathrm{S}_{4}-10$ & -0.58 & 90.5 & -1.32 & 96 & 7.10 & 135.7 \\
\hline 3 & $\mathrm{Utn} / \mathrm{S}_{4}-15$ & 0.22 & 90.5 & 0.88 & 96.5 & 2.82 & 106.7 \\
\hline 4 & $\mathrm{Utn} / \mathrm{S}_{4}-18$ & 2.12 & 92.5 & 2.08 & 96 & -6.14 & 149 \\
\hline \multirow[t]{3}{*}{5} & BIL-113 & -2.38 & 91.5 & -1.92 & 93.5 & -5.48 & 94.6 \\
\hline & $\mathrm{SE}_{\left(g_{i}\right)}$ & 0.654 & - & 0.582 & - & 3.31 & - \\
\hline & $\mathrm{SE}_{\left(g_{i-} g_{j)}\right.}$ & 0.925 & - & 0.823 & - & 4.68 & - \\
\hline
\end{tabular}

DT=Days to tasseling, DS=Days to silking, $\mathrm{PH}=\mathrm{Plant}$ height

Table 3. Cont'd.

\begin{tabular}{|c|c|c|c|c|c|c|c|}
\hline \multirow{2}{*}{$\begin{array}{c}\text { SI. } \\
\text { No. }\end{array}$} & \multirow[t]{2}{*}{ Parents } & \multicolumn{2}{|c|}{ EH (cm) } & \multicolumn{2}{|c|}{ TGW (g) } & \multicolumn{2}{|c|}{ Y (t/ha) } \\
\hline & & GCA & mean & GCA & mean & GCA & mean \\
\hline \multicolumn{8}{|c|}{ Lines } \\
\hline 1 & Pac-60/S $4-3$ & 10.98 & 45.63 & 7.18 & 312.125 & 0.06 & 8.46 \\
\hline 2 & Pac-60/S $4-4$ & 6.10 & 31.1 & -12.636 & 207.76 & -0.10 & 3.59 \\
\hline 3 & $\mathrm{Pac}-60 / \mathrm{S}_{4}-9$ & -3.96 & 37.55 & 17.22 & 359 & -0.55 & 5.93 \\
\hline 4 & $\mathrm{Pac}-60 / \mathrm{S}_{4}-18$ & -3.58 & 36.99 & 1.7 & 334.01 & 0.08 & 9.05 \\
\hline \multirow[t]{3}{*}{5} & $\mathrm{Pac}-60 / \mathrm{S}_{4}-21$ & -9.54 & 46.00 & -0.896 & 296 & 0.61 & 5.68 \\
\hline & $\mathrm{SE}_{\left(g_{i}\right.}$ & 2.288 & - & 25.93 & - & 0.60 & - \\
\hline & $\mathrm{SE}_{(} g_{i-} g_{j)}$ & 3.236 & - & 36.68 & - & 0.85 & - \\
\hline \multicolumn{8}{|c|}{ Testers } \\
\hline 1 & $\mathrm{Utn} / \mathrm{S}_{4}-8$ & 1.84 & 41.5 & 10.82 & 377.09 & 0.22 & 6.25 \\
\hline 2 & $\mathrm{Utn} / \mathrm{S}_{4}-10$ & 1.78 & 47.8 & -33.53 & 385 & 0.60 & 10.85 \\
\hline 3 & $\mathrm{Utn} / \mathrm{S}_{4}-15$ & 1.42 & 35.3 & 27.87 & 254.24 & 0.42 & 4.96 \\
\hline 4 & $\mathrm{Utn} / \mathrm{S}_{4}-18$ & -0.68 & 60.7 & -14.37 & 339.76 & 1.03 & 7.77 \\
\hline \multirow[t]{3}{*}{5} & BIL-113 & -4.36 & 34.8 & 9.21 & 317.12 & 0.99 & 5.89 \\
\hline & $\mathrm{SE}_{\left(g_{i}\right)}$ & 2.288 & - & 25.94 & - & 0.60 & - \\
\hline & $\mathrm{SE}_{\left(g_{i-} g_{j}\right)}$ & 3.236 & - & 36.68 & - & 0.85 & - \\
\hline
\end{tabular}

$* \mathrm{P} \leq 0.05$ and $* * \mathrm{P} \leq 0.01$

$\mathrm{EH}=$ Ear height, $\mathrm{TGW}=1000$ grain weight and $\mathrm{Y}=$ Yield 


\section{Specific combining ability}

Specific combining ability and mean of the crosses for grain yield, its components and other characters are presented in Table 4. In this investigation, none of the crosses showed desirable significant SCA effects for all the characters studied. In case of maize, negative value is expected for days to $50 \%$ tasseling and silking to develop early variety. Significant negative SCA effect also looked for plant height and ear height to develop dwarf variety. However, five crosses Pac-60/ $\mathrm{S}_{4}-9 \times \mathrm{BIL}-113, \mathrm{Pac}-60 / \mathrm{S}_{4}-3 \times \mathrm{Utn} / \mathrm{S}_{4}-18, \mathrm{Pac}-60 / \mathrm{S}_{4}-3 \times \mathrm{Utn} / \mathrm{S}_{4}-$ 15, Pac-60/S $4-9 \times U t n / S_{4}-10$ and Pac-60/S $S_{4}-21 \times B I L-113$ showed non-significant negative SCA effect for both days to tasseling and silking. Four crosses Pac$60 / \mathrm{S}_{4}-21 \times \mathrm{Utn} / \mathrm{S}_{4}-18, \mathrm{Pac}-60 / \mathrm{S}_{4}-21 \times \mathrm{Utn} / \mathrm{S}_{4}-8$, Pac-60/S ${ }_{4}-18 \times \mathrm{Utn} / \mathrm{S}_{4}-15$ and Pac$60 / \mathrm{S}_{4}-9 \times$ BIL-113 showed non-significant negative SCA with lower plant and ear height. Six crosses Pac-60/S $\mathrm{S}_{4}-21 \times \mathrm{Utn} / \mathrm{S}_{4}-15$, Pac-60/S $4-18 \times U \mathrm{Utn} / \mathrm{S}_{4}-15, \mathrm{Pac}-60 / \mathrm{S}_{4-}$ $3 \times \mathrm{Utn} / \mathrm{S}_{4}-18, \mathrm{Pac}-60 / \mathrm{S}_{4}-4 \times \mathrm{Utn} / \mathrm{S}_{4}-18, \mathrm{Pac}-60 / \mathrm{S}_{4}-21 \times \mathrm{Utn} / \mathrm{S}_{4}-10$ and $\mathrm{Pac}-60 / \mathrm{S}_{4^{-}}$ $9 \times \mathrm{Utn} / \mathrm{S}_{4}-8$ had positive SCA with higher grain weight. Though none of the crosses had significant SCA but nine crosses viz. Pac-60/S $4-21 \times$ BIL-113, Pac60/S ${ }_{4}-21 \times U t n / S_{4}-15, \mathrm{Pac}-60 / \mathrm{S}_{4}-18 \times \mathrm{Utn} / \mathrm{S}_{4}-8, \mathrm{Pac}-60 / \mathrm{S}_{4}-3 \times \mathrm{BIL}-113, \mathrm{Pac}-60 / \mathrm{S}_{4}-$ $3 \times \mathrm{Utn} / \mathrm{S}_{4}-18, \mathrm{Pac}-60 / \mathrm{S}_{4}-4 \times \mathrm{Utn} / \mathrm{S}_{4}-18, \mathrm{Pac}-60 / \mathrm{S}_{4}-9 \times \mathrm{Utn} / \mathrm{S}_{4}-15, \mathrm{Pac}-60 / \mathrm{S}_{4}-9 \times \mathrm{BIL}-$ 113 and Pac-60/S $\mathrm{S}_{4}-21 \times \mathrm{Utn} / \mathrm{S}_{4}-10$ had higher yield with positive SCA. In addition, these crosses had root lodging of $0,11.1,65.3,0,0,70.8,12.5,0$ and $0 \%$, respectively and stalk lodging of $0,0,4.2,16.7,15,0,0,16.7$ and $6.3 \%$, respectively.

Table 4. Specific combining ability and mean of the crosses for grain yield and other characters (lodging data were not analyzed) in maize

\begin{tabular}{|c|c|c|c|c|c|c|c|}
\hline \multirow{2}{*}{$\begin{array}{l}\text { Sl. } \\
\text { No. }\end{array}$} & \multirow{2}{*}{ Crosses } & \multicolumn{2}{|c|}{ DT } & \multicolumn{2}{|c|}{ DS } & \multicolumn{2}{|c|}{$\mathrm{PH}(\mathrm{cm})$} \\
\hline & & SCA & mean & SCA & mean & SCA & mean \\
\hline 1. & $\mathrm{Pac}-60 / \mathrm{S}_{4}-3 \times \mathrm{Utn} / \mathrm{S}_{4}-8$ & 0.48 & 86.5 & -0.08 & 91.0 & 5.63 & 180.3 \\
\hline 2. & $\mathrm{Pac}-60 / \mathrm{S}_{4}-3 \times \mathrm{Utn} / \mathrm{S}_{4}-10$ & -0.32 & 84.5 & 0.52 & 90.0 & 2.32 & 182.4 \\
\hline 3. & $\mathrm{Pac}-60 / \mathrm{S}_{4}-3 \times \mathrm{Utn} / \mathrm{S}_{4}-15$ & -0.12 & 85.5 & -1.18 & 90.5 & 1.20 & 177.0 \\
\hline 4. & $\mathrm{Pac}-60 / \mathrm{S}_{4}-3 \times \mathrm{Utn} / \mathrm{S}_{4}-18$ & -1.02 & 86.5 & -0.38 & 92.5 & -2.44 & 164.4 \\
\hline 5. & Pac-60/S $4-3 \times B I L-113$ & 0.98 & 84.0 & 1.12 & 90.0 & -6.70 & 160.8 \\
\hline 6. & Pac- $60 / \mathrm{S}_{4}-4 \times \mathrm{Utn} / \mathrm{S}_{4}-8$ & 0.78 & 90.5 & -0.58 & 93.5 & -11.43 & 160.2 \\
\hline 7. & $\mathrm{Pac}-60 / \mathrm{S}_{4}-4 \times \mathrm{Utn} / \mathrm{S}_{4}-10$ & -0.52 & 88.0 & -0.48 & 92.0 & -9.69 & 167.3 \\
\hline 8. & $\mathrm{Pac}-60 / \mathrm{S}_{4}-4 \times \mathrm{Utn} / \mathrm{S}_{4}-15$ & 0.18 & 89.5 & -0.18 & 94.5 & 3.19 & 175.9 \\
\hline 9. & $\mathrm{Pac}-60 / \mathrm{S}_{4}-4 \times \mathrm{Utn} / \mathrm{S}_{4}-18$ & -1.22 & 90.0 & -0.38 & 95.5 & 16.75 & 180.5 \\
\hline 10. & Pac-60/S ${ }_{4}-4 \times$ BIL-113 & 0.78 & 87.5 & 1.62 & 93.5 & 1.19 & 165.6 \\
\hline 11. & Pac- $60 / \mathrm{S}_{4}-9 \times \mathrm{Utn} / \mathrm{S}_{4}-8$ & 1.98 & 89.0 & 2.62 & 96.0 & 5.17 & 167.9 \\
\hline 12. & $\mathrm{Pac}-60 / \mathrm{S}_{4}-9 \times \mathrm{Utn} / \mathrm{S}_{4}-10$ & -0.82 & 85.0 & -1.78 & 90.0 & -3.74 & 164.4 \\
\hline 13. & $\mathrm{Pac}-60 / \mathrm{S}_{4}-9 \times \mathrm{Utn} / \mathrm{S}_{4}-15$ & 1.38 & 88.0 & 2.02 & 96.0 & -0.56 & 163.3 \\
\hline 14. & $\mathrm{Pac}-60 / \mathrm{S}_{4}-9 \times \mathrm{Utn} / \mathrm{S}_{4}-18$ & -0.52 & 88.0 & -0.68 & 94.5 & 1.00 & 155.9 \\
\hline 15. & Pac-60/S ${ }_{4}-9 \times$ BIL-113 & -2.02 & 82.0 & -2.18 & 89.0 & -1.86 & 153.7 \\
\hline
\end{tabular}




\begin{tabular}{|c|c|c|c|c|c|c|c|}
\hline \multirow{2}{*}{$\begin{array}{l}\text { Sl. } \\
\text { No. }\end{array}$} & \multirow{2}{*}{ Crosses } & \multicolumn{2}{|c|}{ DT } & \multicolumn{2}{|c|}{ DS } & \multicolumn{2}{|c|}{$\mathrm{PH}(\mathrm{cm})$} \\
\hline & & SCA & mean & SCA & mean & SCA & mean \\
\hline 16. & Pac-60/S $S_{4}-18 \times U \operatorname{tn} / S_{4}-8$ & -2.52 & 89.0 & -1.18 & 94.0 & 4.61 & 164.9 \\
\hline 17. & Pac-60/S $\mathrm{S}_{4}-18 \times U \operatorname{Utn} / \mathrm{S}_{4}-10$ & -0.32 & 90.0 & -0.58 & 93.0 & 0.30 & 166.0 \\
\hline 18. & Pac-60/S $\mathrm{S}_{4}-18 \times \mathrm{Utn} / \mathrm{S}_{4}-15$ & -0.12 & 91.0 & 0.22 & 96.0 & -10.22 & 151.2 \\
\hline 19. & Pac-60/S $\mathrm{S}_{4}-18 \times \mathrm{Utn} / \mathrm{S}_{4}-18$ & 1.98 & 95.0 & 1.02 & 98.0 & 1.74 & 154.2 \\
\hline 20. & Pac-60/S ${ }_{4}-18 \times$ BIL-113 & 0.98 & 89.5 & 0.52 & 93.5 & 3.58 & 156.7 \\
\hline 21. & Pac-60/S $\mathrm{S}_{4}-21 \times \mathrm{Utn} / \mathrm{S}_{4}-8$ & -0.72 & 88.5 & -0.78 & 93.0 & -3.97 & 145.9 \\
\hline 22. & Pac-60/S $\mathrm{S}_{4}-21 \times \mathrm{Utn} / \mathrm{S}_{4}-10$ & 1.98 & 90.0 & 2.32 & 94.5 & 10.82 & 166.1 \\
\hline 23. & Pac-60/S $\mathrm{S}_{4}-21 \times \mathrm{Utn} / \mathrm{S}_{4}-15$ & -1.32 & 87.5 & -0.88 & 93.5 & 6.40 & 157.4 \\
\hline 24. & Pac-60/S $\mathrm{S}_{4}-21 \times U \mathrm{Utn} / \mathrm{S}_{4}-18$ & 0.78 & 91.5 & 0.42 & 96.0 & -17.04 & 125.0 \\
\hline 25. & Pac-60/S $4-21 \times B I L-113$ & -0.72 & 85.5 & -1.08 & 90.5 & 3.80 & 146.5 \\
\hline & & 1.46 & - & 1.30 & - & 7.40 & \\
\hline & $\mathrm{SE}_{(S i j-S k l)}$ & 2.07 & - & 1.84 & - & 10.47 & \\
\hline
\end{tabular}

$* \mathrm{P} \leq 0.05$ and $* * \mathrm{P} \leq 0.01$

DT=Days to tasseling, DS=Days to silking, $\mathrm{PH}=$ Plant height

Table 4.cont'd.

\begin{tabular}{|c|c|c|c|c|c|c|c|c|c|}
\hline \multirow{2}{*}{$\begin{array}{c}\text { Sl. } \\
\text { No. }\end{array}$} & \multirow{2}{*}{ Crosses } & \multicolumn{2}{|c|}{$\mathrm{EH}(\mathrm{cm})$} & \multicolumn{2}{|c|}{ TGW (g) } & \multicolumn{2}{|c|}{$\mathrm{Y}(\mathrm{t} / \mathrm{ha})$} & \multicolumn{2}{|c|}{ Lodging } \\
\hline & & SCA & mean & SCA & mean & SCA & mean & Root & Stalk \\
\hline 1. & $\mathrm{Pac}-60 / \mathrm{S}_{4}-3 \times \mathrm{Utn} / \mathrm{S}_{4}-8$ & 7.76 & 87.8 & -43.78 & 389.32 & -0.38 & 11.78 & 25.0 & 7.1 \\
\hline 2. & $\mathrm{Pac}-60 / \mathrm{S}_{4}-3 \times \mathrm{Utn} / \mathrm{S}_{4}-10$ & 3.52 & 83.5 & 44.61 & 433.36 & 0.05 & 11.39 & 3.6 & 0.0 \\
\hline 3. & Pac-60/S $4-3 \times U \operatorname{tn} / S 4-15$ & 2.38 & 82.0 & 5.05 & 455.20 & -1.48 & 10.88 & 3.8 & 19.2 \\
\hline 4. & $\mathrm{Pac}-60 / \mathrm{S}_{4}-3 \times \mathrm{Utn} / \mathrm{S}_{4}-18$ & -2.82 & 74.7 & 2.41 & 410.32 & 1.45 & 12.36 & 0.0 & 15.0 \\
\hline 5. & Pac-60/S 4 -3×BIL-113 & -10.84 & 63.0 & -8.29 & 423.20 & 0.37 & 13.29 & 0.0 & 16.7 \\
\hline 6. & Pac-60/S $\mathrm{S}_{4}-4 \times \mathrm{Utn} / \mathrm{S}_{4}-8$ & -4.86 & 70.3 & -13.68 & 424.88 & -0.74 & 11.27 & 33.3 & 16.7 \\
\hline 7. & $\mathrm{Pac}-60 / \mathrm{S}_{4}-4 \times \mathrm{Utn} / \mathrm{S}_{4}-10$ & -6.40 & 68.7 & -7.32 & 386.88 & -0.86 & 10.33 & 50.9 & 28.6 \\
\hline 8. & Pac-60/S ${ }_{4}-4 \times U$ tn $/ S_{4}-15$ & -2.34 & 72.4 & -34.80 & 420.80 & -0.31 & 11.89 & 54.3 & 0.0 \\
\hline 9. & Pac-60/S ${ }_{4}-4 \times U \operatorname{tn} / \mathrm{S}_{4}-18$ & 12.86 & 85.5 & 45.99 & 459.36 & 2.13 & & 70.8 & 0.0 \\
\hline 10. & Pac-60/S ${ }_{4}-4 \times$ BIL-113 & 0.74 & 69.7 & 9.81 & 446.76 & -0.22 & 12.56 & 61.7 & 2.8 \\
\hline 11. & Pac- $60 / \mathrm{S}_{4}-9 \times \mathrm{Utn} / \mathrm{S}_{4}-8$ & 0.90 & 66.0 & 67.82 & 476.52 & 0.18 & 11.73 & 17.2 & 26.0 \\
\hline 12. & Pac-60/S ${ }_{4}-9 \times U \operatorname{tn} / S_{4}-10$ & 0.96 & 66.0 & -82.03 & 282.32 & -0.16 & 10.58 & 0.0 & 0.0 \\
\hline 13. & Pac-60/S ${ }_{4}-9 \times U \operatorname{tn} / \mathrm{S}_{4}-15$ & 2.52 & 67.2 & -22.31 & 403.44 & 0.43 & 12.18 & 12.5 & 0.0 \\
\hline 14. & Pac-60/S ${ }_{4}-9 \times U$ tn $/ S_{4}-18$ & -2.58 & 60.0 & 19.69 & & -0.68 & 9.62 & 25.0 & 29.2 \\
\hline 15. & Pac-60/S $4-9 \times$ BIL-113 & -1.80 & 57.1 & 16.83 & 423.92 & 0.22 & 12.54 & 0.0 & 16.7 \\
\hline 16. & Pac-60/S $\mathrm{S}_{4}-18 \times \mathrm{Utn} / \mathrm{S}_{4}-8$ & 0.62 & 66.1 & -23.34 & 400.88 & 1.02 & 13.10 & 65.3 & 4.2 \\
\hline 17. & Pac-60/S ${ }_{4}-18 \times U \operatorname{tn} / \mathrm{S}_{4}-10$ & -5.22 & 60.2 & 15.73 & 395.60 & 0.49 & 11.76 & 10.0 & 16.7 \\
\hline 18. & Pac-60/S $\mathrm{S}_{4}-18 \times U \operatorname{tn} / \mathrm{S}_{4}-15$ & -6.06 & 59.0 & 25.49 & 466.76 & 1.22 & 13.50 & 20.2 & 0.0 \\
\hline 19. & Pac-60/S $\mathrm{S}_{4}-18 \times U \operatorname{tn} / \mathrm{S}_{4}-18$ & 3.54 & 66.5 & -15.75 & 383.28 & -2.11 & 8.73 & 92.9 & 0.0 \\
\hline 20. & Pac-60/S ${ }_{4}-18 \times B I L-113$ & 7.12 & 66.4 & -2.13 & 420.48 & -0.62 & 12.23 & 12.5 & 0.0 \\
\hline 21. & Pac-60/S ${ }_{4}-21 \times U \operatorname{tn} / \mathrm{S}_{4}-8$ & -4.42 & 55.1 & 12.98 & 438.00 & -0.09 & 12.62 & 13.9 & 0.0 \\
\hline 22. & Pac-60/S $\mathrm{S}_{4}-21 \times \mathrm{Utn} / \mathrm{S}_{4}-10$ & 7.14 & 66.6 & 29.01 & 409.68 & 0.48 & 12.37 & 0.0 & 6.3 \\
\hline
\end{tabular}




\begin{tabular}{|c|c|c|c|c|c|c|c|c|c|}
\hline \multirow{2}{*}{$\begin{array}{c}\text { Sl. } \\
\text { No. }\end{array}$} & \multirow{2}{*}{ Crosses } & \multicolumn{2}{|c|}{$\mathrm{EH}(\mathrm{cm})$} & \multicolumn{2}{|c|}{$\mathrm{TGW}(\mathrm{g})$} & \multicolumn{2}{|c|}{ Y (t/ha) } & \multicolumn{2}{|c|}{ Lodging } \\
\hline & & SCA & mean & SCA & mean & SCA & mean & Root & Stalk \\
\hline 23. & Pac-60/S ${ }_{4}-21 \times U \operatorname{tn} / \mathrm{S}_{4}-15$ & 3.50 & 62.6 & 26.57 & 468.64 & 0.15 & 13.07 & 11.1 & 0.0 \\
\hline 24. & Pac-60/S $\mathrm{S}_{4}-21 \times U \operatorname{tn} / \mathrm{S}_{4}-18$ & -11.00 & 46.0 & -52.34 & 347.50 & -0.79 & 10.67 & 0.0 & 0.0 \\
\hline 25. & Pac-60/S 4 -21×BIL-113 & 4.78 & 58.1 & -16.22 & 407.20 & 0.26 & 13.74 & 0.0 & 0.0 \\
\hline & $\mathrm{SE}_{(S i j)}$ & 5.12 & & - & - & 1.35 & - & & \\
\hline & $\mathrm{SE}_{(S i j-S k l)}$ & 7.24 & & - & - & 1.90 & - & & \\
\hline
\end{tabular}

$* \mathrm{P} \leq 0.05$ and $* * \mathrm{P} \leq 0.01$

$\mathrm{EH}=$ Ear height, $\mathrm{TGW}=1000$ grain weight and $\mathrm{Y}=$ Yield

\section{Conclusion}

Based on mean performance and GCA effects the lines Pac-60/S $\mathrm{S}_{4}-3$ and Pac$60 / \mathrm{S}_{4}-9$ were identified as a good general combiner for days to tasseling and days to silking, the lines Pac-60/S $4-9$, Pac-60/S $\mathrm{S}_{4}-18$ and Pac-60/S 4 - 21 for shorter plant height and line Pac-60/S $\mathrm{S}_{4} 3$ for grain yield. Furthermore, the tester BIL-113 was identified as a good general combiner for earliness, short plant height and grain yield. These lines and tester could be employed in hybridization program to improve specific trait. Considering lodging, yield performance and other traits the crosses Pac-60/S $\mathrm{S}_{4}-21 \times \mathrm{BIL}-113, \quad \mathrm{Pac}-60 / \mathrm{S}_{4}-21 \times \mathrm{Utn} / \mathrm{S}_{4}-15, \quad \mathrm{Pac}-60 / \mathrm{S}_{4-}$ $21 \times \mathrm{Utn} / \mathrm{S}_{4}-10, \mathrm{Pac}-60 / \mathrm{S}_{4}-9 \times \mathrm{Utn} / \mathrm{S}_{4}-15$ and $\mathrm{Pac}-60 / \mathrm{S}_{4}-9 \times \mathrm{BIL}-113$ showed better performance. These crosses need to evaluate further in wider agro-climatic conditions.

\section{Reference}

Acharya, V. B. and R. Young. 2008. A review of the potential of bio-ethanol in New Zealand. Bulletin of Science Technology Society. 28: 143.

Ahmed, A., M. Amiruzzaman, S. Begum, M. K. Alam, and M. M. Rohman. 2015. Analysis of combining ability and gene action of field corn through line $\times$ tester method. J. Expt. Biosci. 6(2): 61-66.

Ali, G., I. Ahmed, S. A. Dar and A. M. Iqbal. 2012. Combining ability analysis for yield and its component traits in high altitude maize (Zea mays L.) inbreds. Adv. in Life Sci. 1 (1): 66-69.

Amin, M. N., M. Amiruzzaman, A. Ahmed and M. R. Ali. 2014. Evaluation of inbred lines of maize (Zea mays L.) through line $\times$ tester method. Bangladesh J. Agril. Res. 39(4): 675-683.

Ceyhan, E. 2003. Determination of some agricultural characters and their heredity through line $\times$ tester method in pea parents and crosses. Selcuk University, Graduate School Nat. Applied Science, p.103.

Chandel, U. and B. S. Mankotia. 2014. Combining ability in local and CIMMYT inbred lines of maize (Zea mays L.) for grain yield and yield components using line $\times$ tester analysis. SABRAO J. Breed. and Gen. 46 (2): 256-264.

DAE. 2017. Krishi Diary. Agricultural Information Service. 
EL-Hosary, A. A. A. 2014. Relative value of three different testers in evaluating combining ability of new maize inbred lines. International J. plant Breed. and Gen. 8(2):57-65.

Hallauer, A. R. 1975. Relation of gene action and type of tester in maize breeding procedures. Proc. Ann. Corn \& Sorghum Res. Conf. 30: 150-165.

Hallauer, A. R. and J. B. Miranda. 1988. Quantitative genetics in maize breeding. 2nd ed. Iowa State Univ. Press. Ames. pp. 64-71.

Joshi, V. N., R. B. Dubey and S. Marker. 2002. Combining ability for polygenic traits in early maturity hybrids of maize (Zea mays L.). Indian J. Plant Breed. Genet. 62(4): 312-315.

Kamara, M. M., S. Ibrahim, I. S. El-Degwy and H. Koyama. 2015. Estimation combining ability of some maize inbred lines using line $\times$ tester mating design under two nitrogen levels. Australian. J. Crop. Sci. 8(9):1336-1342.

Kanagarasu, S., G. Nallathambi, and K. N. Ganesan. 2010. Combining ability analysis for yield and its component traits in maize (Zea mays L.). Electronic J. Pl. Breed. 1(4): 915-920.

Kempthorne, O. 1957. An Introduction to Genetic Statistics. New York: John Wiley \& Sons, Inc. London: Chapman \& Hall Ltd. p. 458-471.

Kumar, B. S., M. Prakash, G. Sathyanarayanan, and S. Padmavathi. 2012. Studies on combining ability and heterosis through line $\times$ tester analysis in maize (Zea mays L.). Crop Res., 43 (1/2/3): 153-157.

Kumar, G. P., V. Narsimha, S. Reddy, k. Sudheer and P. V. Rao. 2014. Combining ability studies in newly developed inbred lines in maize (Zea mays L.). Intl. J. P. Ani. and Env. Sci. 4 (4): 229-234.

Liaqat, S., H. U. Rahman, A. Ali, N. A. Bazai and M. M. Tahir. 2015. Combining ability estimates from line $\times$ tester mating design in maize (Zea mays L.). Acad. Res. J. Agri. Sci. Res. 3(4):71-75.

Menz, M. A., A. R. Hallauer and W. A. Russell. 1999. Comparative response of two reciprocal recurrent selection methods in BS21 and BS22 maize populations. Crop Sci. 39(1): 89-97.

Rahman, H., A. Ali, Z. Shah, M. Iqbal, M. Noor and M. Amanullah. 2013. Line $\times$ tester analysis for grain yield and yield related traits in maize variety sarhad-white. Pak. $J$. Bot. 45(SI): 383-387.

Rawlings, J. O. and D. L. Thompson. 1962. Performance level as criterion for the choice of maize testers. Crop Sci. 2: 217-220.

Sharma, S. M., S. Narwal, R. Kumar and S. Dass. 2004. Line $\times$ tester analysis in maize (Zea mays L.). Forage Res. 30: 28-30.

Singh, D. N. and I. S. Singh. 1998. Line $\times$ tester analysis in maize. J. Res. Birsa Agric. Univ., 10(2): 177-182

Suneetha, Y., J. R. Patel and T. Srinivas. 2000. Studies on combining ability for forage characters in maize (Zea mays L.). Crop Res. 19(2):226-270.

www.worldcornproduction.com (accessed on 27 March, 2017). 